\title{
GPS, Accelerometer, and Smartphone Fused Smart Sensor for SHM on Real-Scale Bridges
}

\author{
G. Michel Guzman-Acevedo, ${ }^{1}$ G. Esteban Vazquez-Becerra, ${ }^{1}$ Jesus R. Millan-Almaraz, ${ }^{2}$ \\ Hector E. Rodriguez-Lozoya, ${ }^{3}$ Alfredo Reyes-Salazar $\mathbb{D}^{3},{ }^{3}$ J. Ramon Gaxiola-Camacho $\mathbb{D}^{3},{ }^{3}$ \\ and Carlos A. Martinez-Felix ${ }^{1}$
}

${ }^{1}$ Department of Earth and Space Sciences, Autonomous University of Sinaloa, 80040 Culiacan, Mexico
${ }^{2}$ Department of Physics and Mathematics, Autonomous University of Sinaloa, 80040 Culiacan, Mexico
${ }^{3}$ Department of Civil Engineering, Autonomous University of Sinaloa, 80040 Culiacan, Mexico

Correspondence should be addressed to J. Ramon Gaxiola-Camacho; jrgaxiola@uas.edu.mx

Received 7 March 2019; Revised 4 June 2019; Accepted 10 June 2019; Published 23 June 2019

Academic Editor: Reza Akhavian

Copyright (c) 2019 G. Michel Guzman-Acevedo et al. This is an open access article distributed under the Creative Commons Attribution License, which permits unrestricted use, distribution, and reproduction in any medium, provided the original work is properly cited.

\begin{abstract}
During the last two decades, Global Positioning System (GPS) geodetic-grade receivers and accelerometers have been implemented in Structural Health Monitoring (SHM). Most recently, the use of sensors integrated in smartphones has been evolving. Although some of their capabilities are validated for small and local structures, there is a gap in knowledge about the use of sensors embedded in smartphones and other electronic devices for SHM of complex structures as bridges. To contribute in this area, this paper demonstrates the application of GPS receivers, accelerometers, and smartphones, integrating a smart sensor for the SHM of bridges. In order to validate its capabilities, the alternative smart sensor is used to study a particular bridge with vibration problems. Semistatic and dynamic displacements are obtained by means of GPS measurements. Accelerations in three directions of the bridge are determined using the accelerometer and the smartphone. Based on the results of the alternative smart sensor, inappropriate structural behavior is detected in the vertical direction of the bridge. In addition, dynamic characteristics are extracted using the smart sensor applying the Fast Fourier Transformation (FFT) and periodogram to the structural responses. As a result, it is verified the applicability of the fused smart sensor for SHM on real-scale bridges.
\end{abstract}

\section{Introduction}

Since two decades ago, both geodetic-grade GPS (Global Positioning System) receivers and commercial accelerometers have been used in several studies related to Structural Health Monitoring (SHM) [1-9]. The results of these investigations demonstrated that they are efficient and accurate devices for SHM of roads, dams, buildings, bridges, etc. However, there are some deficiencies associated to them. For example, it is well known that GPS signal is generally affected when it passes through the different layers of the atmosphere (ionosphere and troposphere). In addition, some objects surrounding the GPS antenna environment also produce multipath effects. In consequence, the GPS signal reflects to the receiver, i.e., the GPS antenna obtain both direct and indirect signals [10]. As an alternative, it is documented in the literature that SHM can be performed using commercial accelerometers $[11,12]$. Nevertheless, they present reduced precision in real time. Furthermore, static and semistatic displacements cannot be directly obtained [3], i.e., a double integration must be implemented for calculating displacement from acceleration time series [11] producing differences from decimeter to even meter level with respect to real values [13]. Some researchers have proposed to integrate GPS devices and accelerometers in implementing SHM $[2,6,14]$, but one of the biggest issues is that both instruments are very expensive. This problem opens an opportunity to implement different methodologies or even new instruments to collect data. One option may be to use sensors that are generally integrated in smartphones; these 
devices have been studied, and it is demonstrated, in certain way, that they are reliable in terms of accuracy and efficiency for relatively small and local structures $[15,16]$. For example, Zhao et al. [17] validated the implementation of smartphones in extracting force measurement of cables in bridges. Particularly, they proposed an easy-to-handle, convenient, low cost, time-saving software in SHM of bridges that can be operated in smartphones [18]. In addition, Ding et al. [19] documented a bridge inspection and management system using smartphones. Such a system was demonstrated to be an opportunity for management and inspection of bridges located in rural and mountainous areas where the access to them may be very difficult. Furthermore, very recently, it was reported in the literature the validation of smartphones particularly under seismic loading using shaking tables for the excitation of small frames [20]. However, to increase the state of the art in this area, additional investigations must be performed related to the use of smartphones in SHM of complex structures under serviceability conditions in real time. Furthermore, measurements of the structural responses of such structures should be performed considering the real scale of them. At present, smartphones contain elements for storing data, RAM, and advanced microprocessors, and the recorded information can be transferred using mobile Internet, WiFi, or Bluetooth connectivity. In addition to integrated accelerometer, smartphones possess other sensors such as gyroscope and magnetometer [15]. Hence, the main difficulty about using GPS receivers, accelerometers, and smartphones in SHM of complex structures as bridges may be related to the next three questions: (1) do GPS receivers, accelerometers, and smartphones provide appropriate accuracy in structural response measurements of real-scale bridges?, (2) can GPS receivers, accelerometers, and smartphones be complemented to each other?, and (3) can GPS receivers, accelerometers, and smartphones be utilized to integrate an alternative smart sensor for the SHM of bridges? To address the above three issues, the main objective and contribution of this paper to the literature is that it will demonstrate the sensing capabilities of GPS receivers, accelerometers, and smartphones in terms of an alternative fused smart sensor, implementing its application with the study of a bridge structure with serious vibration problems.

\section{Fused Smart Sensor-Integration of GPS Receivers, an Accelerometer, and a Smartphone}

In this research, the following instruments are used to propose an alternative smart sensor for SHM of bridges: (1) geodetic-grade GPS receivers, (2) a commercial accelerometer, and (3) a smartphone. The above instruments are used to collect displacement and acceleration data, and after proper evaluation and signal processing, they are used for interpretation of the structural behavior of a real-scale bridge.

2.1. Geodetic-Grade GPS Receivers. The use of GPS receivers related to SHM has been implemented in several investigations [1, 3-9], resulting that GPS is an advanced tool for this kind of purposes. The main advantage of GPS is its capability of measuring directly displacements without the requirement of double integration of accelerations; generally necessary in other devices. GPS receivers can determine dynamic, static, semistatic displacements, and characteristic frequencies of structures [7]. Furthermore, modern geodetic-grade GPS receivers allow data collection with a sampling rate of 50 to $100 \mathrm{~Hz}$ [21] providing high precision and automatic operation, and they do not need a line of sight between receivers. On the other hand, GPS may present some disadvantages [2,6] that can be reduced by applying different methodologies related to signal processing techniques as several filters that significantly improve the results. Such filters will be discussed later in this paper. For the smart sensor presented in this research, two similar geodetic-grade GPS receivers as illustrated in Figure 1 are used for the SHM on real-scale bridges.

The brand of the GPS receiver used is Septentrio model AsteRx-U with PolaNt-x MF antenna. The second author of this paper contacted Septentrio Inc. for the evaluation of such an equipment. Some of the main specifications of these devices are 544 channels for tracking satellite constellation of GPS, GLONASS, GALILEO, BEIDOU, IRNSS and QZSS; RTK horizontal and vertical accuracy of $0.6 \mathrm{~cm}+0.5 \mathrm{ppm}$ and $1 \mathrm{~cm}+1 \mathrm{ppm}$, respectively. According to the manufacturer, it obtains data from L1, L2, and L5 carrier frequencies just considering GPS constellation. In addition, their maximum sampling frequency is $100 \mathrm{~Hz}$. Therefore, based on the Nyquist-Shannon theorem [22], the effective bandwidth of this receiver ranges from 0 to $50 \mathrm{~Hz}$. Even though the receiver employed can record data from different satellite constellations, in this paper, the authors only considered the GPS constellation.

2.2. Commercial Accelerometer. Same as GPS receivers, commercial accelerometers have been tested in SHM $[2,6]$. For example, it was documented by Celebi [11] that structural response collected using accelerometer produced positive results, when earthquake or wind loading excites structures. Acceleration data have been used for evaluation, analysis, or even design, finding a relationship between damage and reaction and developing better code provisions. In addition, in comparison with GPS receivers, accelerometers provide higher sampling frequency, which is one of their main advantages. Hence, it can be obtained better information and more data about dynamic characteristics of the structure under consideration. For example, some commercial accelerometers can obtain dynamic information from structures with a natural frequency greater than $1,000 \mathrm{~Hz}$ [23]. The rate of sampling frequency indicates the quality of the collected behavior. For high sampling rates, the resolution of the measured movement improves significantly. Moreover, according to Kaloop [22]; the Nyquist theorem must be satisfied:

$$
f_{\mathrm{s}} \geq 2 f_{0}
$$

where $f_{\mathrm{s}}$ is the sampling frequency and $f_{0}$ is the fundamental frequency of the structure being monitored. 


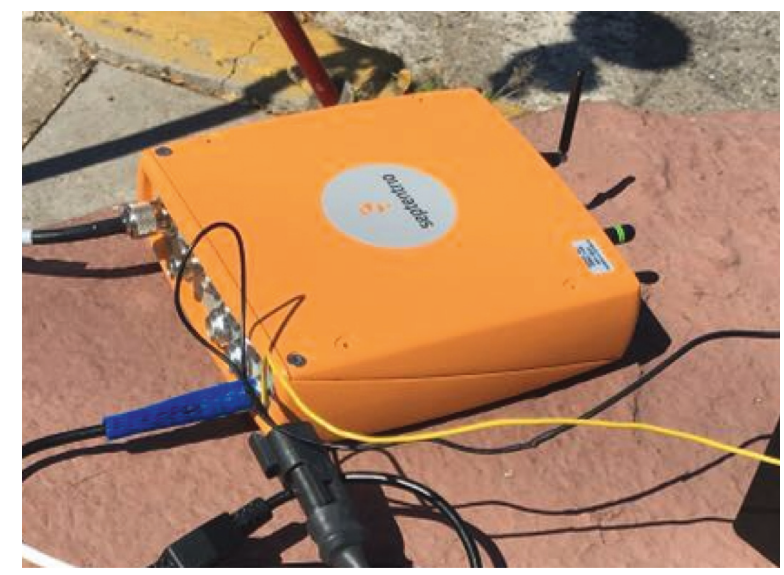

(a)

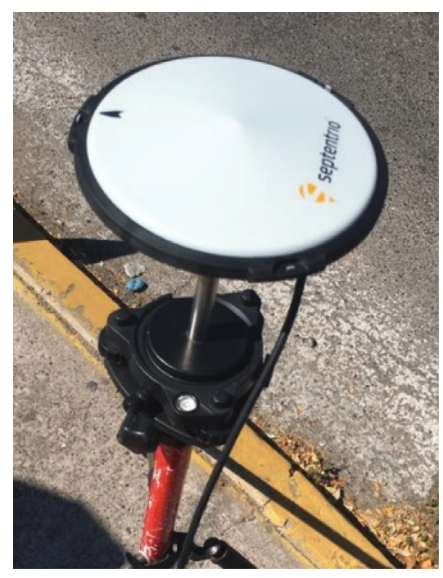

(b)

FIGURE 1: GPS sensors used: (a) receiver unit; (b) antenna.

Another benefit of accelerometers with respect to GPS receivers is that they are less affected by external conditions, i.e., accelerometers are more independent. In some cases, the accelerometer can improve the structural monitoring process because of the quantity of data acquired [6]. For the smart sensor presented in this paper, the commercial accelerometer used is an ETNA high-dynamic range strong motion accelerometer (see Figure 2). Some of the main specifications of this accelerometer are 18 bits of resolution with $108 \mathrm{~dB}$ dynamic range, cost-effective solution that can satisfy today's most demanding applications, multitasking operating system that allows simultaneous data acquisition and interrogation, timing accuracy to 0.5 milliseconds due to synchronized sampling with optional GPS timing system, remote alerting capability for system event or autodiagnostic failure, interconnectivity with other Altus family recorders for common triggering, and shared GPS (optional), common user interface, file format, and support tools with other Altus family recorders.

2.3. Brand-New Smartphones. As previously mentioned, modern smartphones have different kind of sensors such as gyroscopes, magnetometers, and accelerometers. However, because of the scope and objectives of this paper, only the integrated accelerometer is evaluated. Several investigations have examined such a sensor incorporated in smartphones, for example, its performance has been demonstrated with respect to commercial accelerometers for small prototypes [15]. The results of both instruments seem to be very similar in time and frequency domain. However, there is a gap of knowledge in the use of sensors embedded in smartphones in the SHM of infrastructure as bridges, considering their real scale. As previously mentioned, one of the main objectives of this paper is to present an alternative to the above concern integrating a smart sensor. To implement smartphones in SHM, some issues must be addressed such as how to preprocess the information reported by accelerometer smart sensor, how to fix the smart sensor to the structure, and problems related to the battery's lack of energy [16]. On the other hand, since sensors are perfectly embedded in smartphones, they are portable, friendly, and easy to handle, i.e., anyone can use them without a background knowledge about their physics, and they are less expensive than GPS receivers and commercial accelerometers, respectively. The model of smartphone employed in this research to be integrated in the smart sensor is Samsung Galaxy S8+ (see Figure 3). The main characteristics of this smartphone are size of $159.5 \times 73.4 \times 8.1 \mathrm{~mm}$, weight $0.173 \mathrm{~kg}$, and Qualcomm Snapdragon 835 processor, it is powered by the octa-core Exynos 8895 processor $2.35 \mathrm{GHz}$ (Quad Core) $+1.9 \mathrm{GHz}$ (Quad), 64 bit and technology of 10 nanometers, 4GB of RAM, storage of $64 \mathrm{~GB}$ and operative system Android 7.0 (Nougat), and accelerometer LSM6DSL (resolution of $0.0023956299 \mathrm{~m} / \mathrm{sec}^{2}$ ). To extract acceleration time series, the embedded accelerometer from the S8+ smartphone was utilized as well as an App named Accelerometer analyzer (See Figure 3(b)). This free App was created by Mobile Tools [24]. It will be demonstrated how this App is an excellent tool to record acceleration data using smartphones. Furthermore, the App plots the graphics in real time and stores the data in a text file for postprocessing.

2.4. Fused Smart Sensor. The above three devices are integrated to form the alternative smart sensor as illustrated in Figure 4 . They are mounted in a special mechanic steel structure developed to hold the three of them: (1) GPS receiver, (2) accelerometer, and (3) smartphone. The supporting structure consists of 3 levels, where each plate supports an instrument. On the top is mounted the GPS antenna, this plate allows GPS antenna to have a correct orientation to North. Below, in the middle level, the commercial accelerometer is located. At the lowest plate, the smartphone is properly fixed.

\section{Calculation of Displacements Using GPS Receivers}

As will be discussed later in this paper, when bridges are being monitored, two deformations need to be considered: 


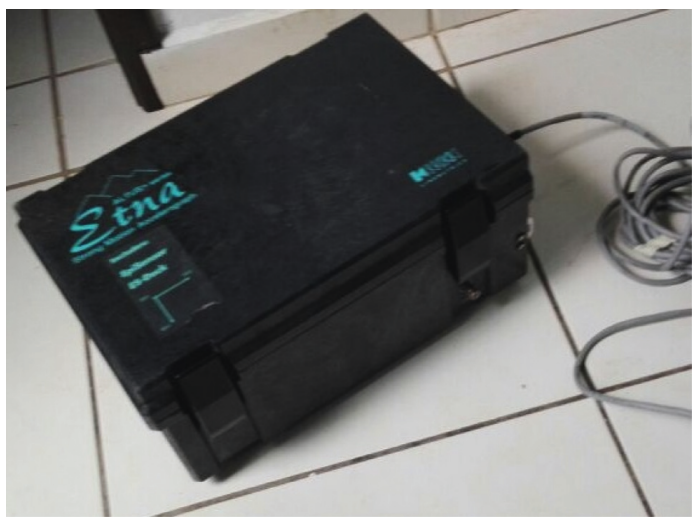

(a)

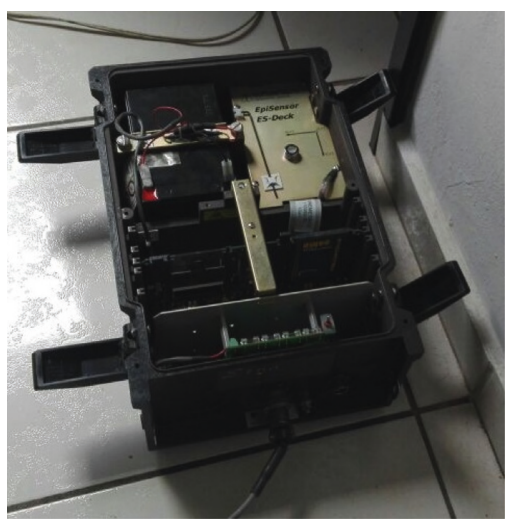

(b)

FIgURE 2: ETNA accelerometer: (a) outside view; (b) interior view.

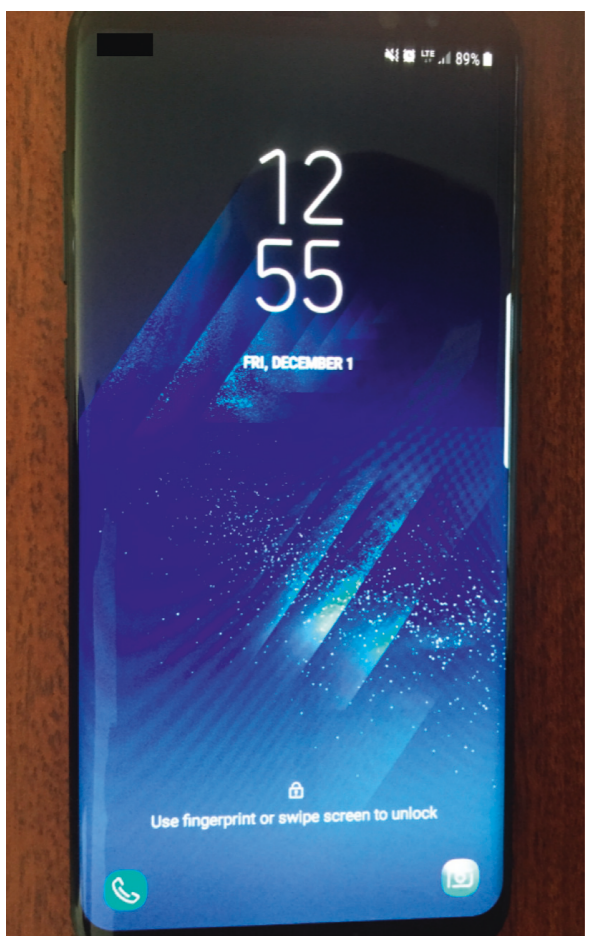

(a)

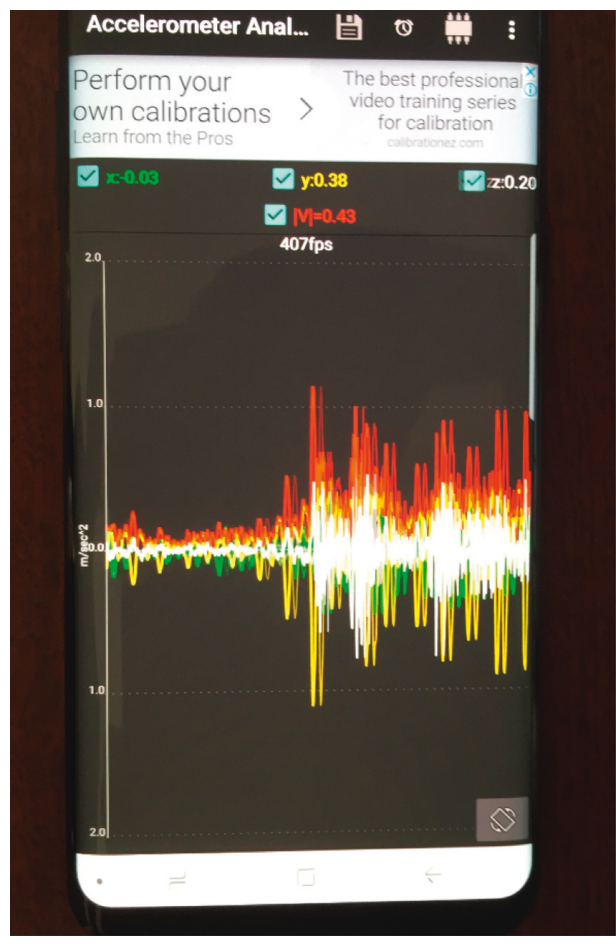

(b)

Figure 3: Samsung Galaxy S8+: (a) general view; (b) App used for acceleration measurement.

(1) short- and (2) long-term periods. Hence, it is crucial to use an instrument that can measure both movements at the same time. Short- and long-term deformations are produced by different loading conditions of the bridge, but in terms of risk, deformations related to short period seems to be more relevant [25]. Before calculating the semistatic and dynamic displacements, the raw GPS time series (output data from the GPS software) formed by coordinates from the World Geodetic system 84 (WGS84) should be transformed to apparent displacement time series. The purpose of this step is to obtain the GPS data around its mean value as follows [21]:

$$
A_{i}=R_{i}-\frac{1}{n} \sum_{i=1}^{n} R_{i},
$$

where $A_{i}$ is the apparent displacement, $R_{i}$ is the raw GPS data, $n$ is the number of sampling points, and $i=1, \ldots, n$.

To study the structural behavior of bridges as accurate as possible using GPS data, several researchers recommended to use the Moving Average and Chebyshev filters over the apparent displacement time series to calculate semistatic and dynamic displacements, respectively $[9,21,26]$. The use of such filters is justified next. 


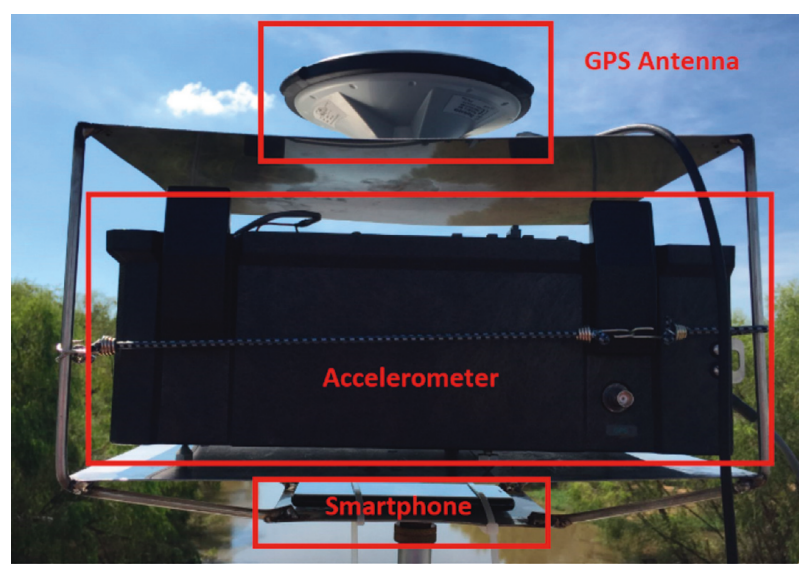

Figure 4: Smart sensor.

3.1. Semistatic Displacements Using Moving Average Filter. Apparent displacement is formed by short- and long-period components. The long component has a slow movement, long wavelength, and low frequency. It is produced by some effects such as foundation settlement, deck creep, thermal expansion/contraction, and loss of prestress [25]. It is reported in the literature that long-period component expresses the semistatic displacement [27], which can be used to evaluate the movement of the bridge [22]. It can be calculated by applying the moving average filter with a window of 41 samples [26]. One problem that must be faced for determining semistatic displacement from GPS data is that long-period component presents colored noise (chaotic behavior) produced by environmental bias (multipath, ionospheric, and tropospheric delay) and instrumental noise (noise produced by each GPS receiver) [28]. Therefore, it is important to take care about how the filter is used. The moving average filter presents a good performance in time domain. Consequently, it can be used for smoothing time series. It takes a specific number of elements from the time series based on the window's size, beginning in the first element, and their average is calculated. Then, it takes the same number of elements, but starting in the second element for calculating their average and so on. The moving average filter is given as [29]

$$
Y[i]=\frac{1}{M} \sum_{j=0}^{M-1} x[i+j],
$$

where $x$ is the input signal, $Y$ is the output signal, and $M$ is the size of window.

3.2. Dynamic Displacements Using Chebyshev Filter. To calculate the short period component representing the dynamic displacement of the structure under consideration, a high-pass filter should be applied to the apparent displacement time series. The short period component is a fast movement with high frequency and short wavelength. This movement is generally produced by traffic, people, earthquake activity, and so on [25]. To clarify this concept, the measured displacement of a bridge using GPS can be expressed as [30, 31]

$$
Y(n)=M(n)+D(n)+N(n),
$$

where $Y(n)$ is the apparent displacement, $M(n)$ represents the long period interferences (environment delays), $D(n)$ is the dynamic displacement of the bridge, and $N(n)$ is the noise.

It is documented in the literature that dynamic displacements can be used to understand the structural performance of bridges [32]. The filter used in this article to extract dynamic displacements is the Chebyshev type I, and it has been tested by some scholars [9, 21, 26, 31]. Chebyshev type I filter can be expressed as [30]

$$
G_{n}(w)=\left|H_{n}(j w)\right|=\frac{1}{\sqrt{1+e^{2}+T_{n}^{2}\left(w / w_{0}\right)}}
$$

where $\left|H_{n}(j w)\right|$ is the transfer function with an absolute value equal to the gains as an angular function of frequency $w$ of order $n ; e$ is the ripple factor; $T$ is the Chebyshev polynomial order; and $w_{0}$ is the cut-off frequency. This filter is characterized by having the ripple in the passband.

\section{Calculation of Displacements Using Acceleration Records}

There are alternative methodologies in the literature for calculating displacement using acceleration records $[6,14]$; that is, to obtain displacements from acceleration time series, a double integration of them is required. However, the problem is how to select the method that provides better results or the one losing less information, i.e., the most efficient and accurate. Obtaining displacement from acceleration records is a difficult process. It introduces bias creating a difference from decimeter to even meter level between direct and indirect results [13]. This process is described in terms of four steps according to Feng et al. [16]. Firstly, a band pass filter needs to be implemented to remove noise. Secondly, a double integration is applied as

$$
s(t)=s_{0}+v_{0} x t+\int_{0}^{t}\left(\int_{0}^{t} a(t) d t\right) d t,
$$

where $s(t)$ is the displacement; $a$ is the acceleration; $s_{0}$ is the initial position; and $v_{0}$ is the initial velocity.

Thirdly, the initial values of position and velocity are set equal to zero. Finally, the linear trend must be removed [14]. Hence, the results of this process represent the dynamic displacement. Unfortunately, because the velocity and position are supposed to be zero, the semistatic and static displacements are lost [6]. In this process is essential to use a correct band pass filter because noise is removed per it. For this purpose, there are several alternatives. For example, it can be used the Discrete Fourier Transform (DFT), a Finite Impulse Response (FIR) filter, or an Empirical Model Decomposition (EMD) filter. The first of them (DFT filter) provides a no complex structure and balance [14]. On the other hand, EMD filter generates a set of $N$ functions from 
the input signal. The resulted set of functions from EMD is defined as [14]:

$$
a(t)=\sum_{n=1}^{N} C_{n}(t)+r_{n}(t),
$$

where $a(t)$ is the input signal, $r_{n}(t)$ is the residue, and $C_{n}(t)$ is the $n^{\text {th }}$ function which is named intrinsic mode functions (IMF). The EMD filter is expressed as [14]

$$
a_{b}(t)=\sum_{n=q}^{r} C_{n}(t),
$$

where

$$
1<q<r<n .
$$

Hence, the above expressions can be used to calculate dynamic displacements from acceleration time histories recorded by smartphone and accelerometer, respectively.

\section{Application of GPS Receivers, Accelerometers, and Smartphones in SHM}

As previously documented, several are the advantages and disadvantages of GPS receivers, accelerometers, and smartphones in SHM. Both GPS receivers and commercial accelerometers have limitations that can be addressed using certain methodologies. For example, integrating GPS and commercial accelerometer, the combined result may recover lost data, e.g., missing measurements from GPS. This integration may solve the lack of semistatic and static components. The redundancy of both systems (GPS and accelerometers) provides a good performance in SHM [6]. In general, it is reported in the literature that high-frequency displacements may be obtained from accelerometers and low frequency ones from GPS. The reason of considering low frequencies from GPS and high ones from accelerometers is that some scholars [14] demonstrated that GPS is a helpful tool for measuring static and semistatic behaviors (low-frequency displacements), but it experiences problems related to collecting data of dynamic displacements from structures vibrating with high frequencies. On the other hand, accelerometer records dynamic responses very accurately without low-frequency components. Therefore, it is reported in the literature that accelerometers and GPS make a reasonable complement to each other $[2,6,14]$. Unfortunately, GPS receivers and accelerometers are expensive instruments. Commonly, the acceleration is recorded using commercial accelerometers. However, the improved quality of acceleration sensors integrated in smartphones provides a feasible alternative. Generally, their level of accuracy may be lower than a commercial accelerometer, but it is reported in the literature that their results are very similar between them for small structural systems $[15,16]$. The contribution of this article to the literature is that accelerometer sensors of smartphones are used, together with other devices, to form a smart sensor to be used in the SHM of a reinforced concrete bridge in real time. Then, a parametric analysis is performed with respect to GPS receivers and commercial accelerometers.

\section{Dynamic Characteristics of Structures}

Before proceeding further with the discussion, dynamic characteristic of structures must be introduced. Structures are designed to resist different types of loading conditions. They must have the capacity to reliably transmit loadings from the applied point to their foundations, when this is happening, movements may be produced in structures as a response. Movements are generated following several frequencies depending mainly on the degrees of freedom of the structure, one of the most important frequencies is the natural or fundamental frequency [33]. To analyze frequencies is necessary to change the analysis from time to frequency domain generally using the Fast Fourier Transformation (FFT). In general sense, FFT is a computational complexity improvement of DFT that separates a complex signal into a set of sinusoidal ones, where each signal has a corresponded frequency and potential. Similarly, there are other techniques such as periodogram. It is welldocumented that both FFT and periodogram algorithms can be utilized for analyzing movements of bridge structures on frequency domain [34]. Because FFT is a well-known algorithm, its mathematical formulation is not described here. On the other hand, periodogram equation can be observed in Equation (10) where $x(t)$ represents the time domain input signal, $X(f)$ is its Fourier transform, and $X p(f)$ is the output periodogram which is calculated as the Fourier transform of the autocorrelation function from $x(t)$. It also can be alternatively calculated as the multiplication between $X(f)$ and its conjugated counterpart as [34]

$$
X p(f)=F\{x(t) * x(-t)\}=X(f) \cdot X^{*}(f) .
$$

\section{Structural Description of the Analyzed Bridge}

The analyzed structure in this paper using the fused smart sensor is the Juarez Bridge (see Figure 5). Such a structure is located in Culiacan, Mexico. It is a reinforced concrete bridge with an approximate length of 200 meters. This is an important civil infrastructure of the city. Some important findings about the structural performance of this bridge were documented earlier by Vazquez-Becerra et al. [26]. It was reported that the bridge presented deformations in average of about $8 \mathrm{~cm}$ in the vertical direction, representing an undesirable structural behavior. In the above research, maximum displacements were recorded at the ZEN2 station (see Figure 5(b)) which was reported to be the most affected location over the bridge, mainly, because several vehicles are stopped by a traffic light close to it. Considering displacements with respect to the vertical direction, the calculated average of probability of failure based on comparing semistatic displacement with AASHTO (American Association of State Highway and Transportation Officials) deflection limits was about $40 \%$. In addition, according to Vazquez-Becerra et al. [26], the Juarez Bridge's probability of failure and its maximum displacements were calculated by the average displacements extracted via GPS receivers located at critical points of the bridge. However, they only used GPS receivers to extract the performance of the structure 


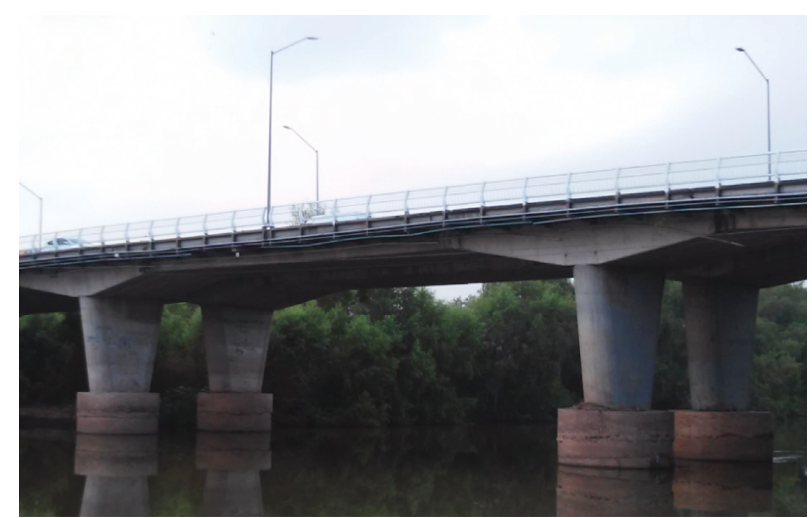

(a)

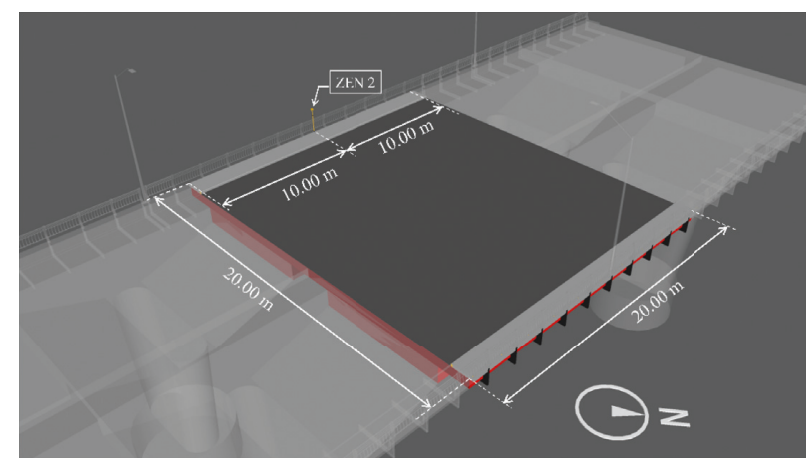

(b)

FIgURE 5: Juarez Bridge: (a) lateral view; (b) ZEN2 GPS location.

under normal loading conditions. On the other hand, for the investigation presented in this paper, the GPS performance was improved by adding acceleration data using commercial accelerometer and smartphone, respectively. The combination between GPS and commercial accelerometers has been developed and reported in the literature $[2,14,23]$. However, very few information is documented in the literature about using sensors embedded in smartphones to record acceleration of real-scale bridges. Consequently, if results obtained via smartphone for bridge structures are viable, this will provide a great advancement in SHM of bridges because price and time would be drastically reduced in extracting their structural performance in real time.

\section{Measurement Campaign Using Fused Smart Sensor}

For this study, the measurement campaign was developed at the ZEN2 station (see Figure 5(b)) because it was previously documented by Vazquez-Becerra et al. [26] that this location presented the worst performance with respect to average displacements and probability of failure. Additionally, these measurements were developed on Monday since this day presented the highest probability of inappropriate structural behavior. The structural assessment of the bridge was developed simultaneously in terms of the smart sensor, integrating GPS receivers, accelerometer, and smartphone. The process is comprehensively described in this section.

8.1. GPS Receivers Campaign. The GPS measurement methodology employed was the kinematic relative positioning, which involves two stations measuring simultaneously, one in movement (the one located on the bridge) and the other one static (outside of the bridge as static reference) [10]. The stable-based reference station used is the $373 \mathrm{H}$ station; this station is located near to the bridge. It is part of the active national geodetic network from the Mexican National Institute of Statistics and Geography (INEGI in Spanish). Figure 6(a) shows the position of station $373 \mathrm{H}$ with respect to the ZEN2 station. The length of the baseline (from ZEN2 to $373 \mathrm{H}$ ) is approximately 120 meters. The displacement measurement lasted 500 seconds, using a sampling rate of $100 \mathrm{~Hz}$ when the Juarez Bridge was subjected to in-service traffic loading conditions. Figures 6(b) and 6(c) illustrate the ZEN2 and $373 \mathrm{H}$ stations, respectively. They were monitored during the GPS displacements campaign.

Previously to postprocessing the collected data, raw GPS data was debugged by Teqc (translation, editing, and quality check) [35]. The preprocessing consists in insulate GPS data and perform a quality check. The latest step is very interesting considering that it provides an understanding of the condition of the GPS receiver's position in terms of Multipath (MP), visible satellites, and signal to noise ratio (S). For this case, the results demonstrate that the rover reports multipath values of MP1RMS $=0.0478 \mathrm{~m}, \mathrm{MP} 2 \mathrm{RMS}=0.0119 \mathrm{~m}, 19 \mathrm{GPS}$ satellites with observations, $S 1=7.01$, and $S 2=4.49$; for the reference station, MP1RMS $=0.0428 \mathrm{~m}, \mathrm{MP} 2 \mathrm{RMS}=0.0114$, $S 1=7.12$, and $S 2=4.49$; the values of both receivers are very similar due to the short distance between them. On the other hand, because relative positioning with double differencing (DD) over a short baseline (less than 10 kilometers of length) was used, some error sources were either reduced or dropped: for example, clock bias from the satellite-receiver and atmospheric effects (ionospheric and tropospheric bias) because both receivers are generally affected by the same conditions [36]. Additionally, this positioning technique makes possible to achieve a good performance using commercial software for processing raw GPS data, which can be seen in most of investigations related to the use of GPS receivers in SHM [7, 21, 28, 31, 32]. However, due to the excessive amount of data, the software considered to process all the information is the GAMIT GLOBK TRACK developed by the MIT (Massachusetts Institute of Technology). This software processes GPS data in kinematic mode. The main specifications of GPS data processing included $15^{\circ}$ cut-off angle, precise final orbits disseminated by IGS (International GNSS Service), mode air (used for high sample), National Geodetic survey antenna calibration parameters, and others.

8.2. Accelerometer Campaign. As previously discussed, during the 500 seconds measurement session, one commercial accelerometer was utilized. This device was 


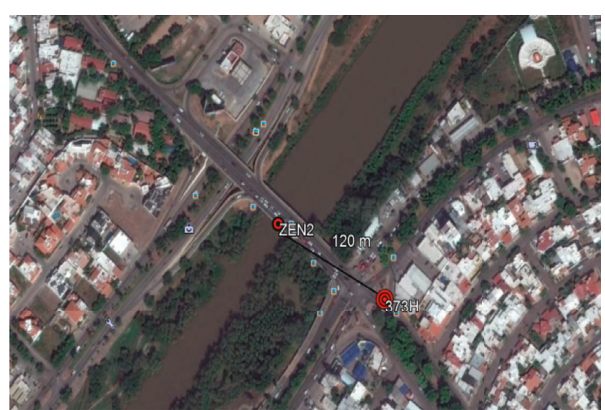

(a)

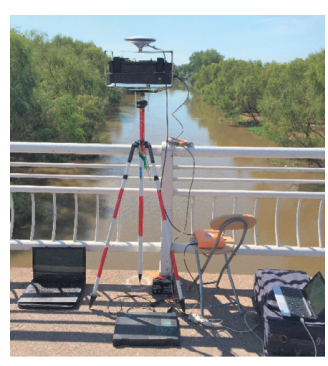

(b)

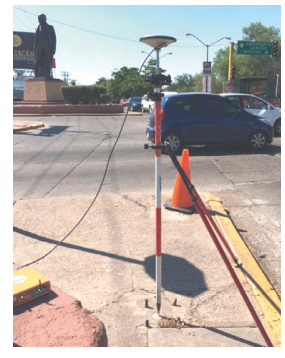

(c)

Figure 6: GPS campaign: (a) location of ZEN2 and 373H; (b) station ZEN2; (c) station 373H.

recording acceleration at a rate of $100 \mathrm{~Hz}$. The accelerometer was perfectly fixed to present the same physical center as the other devices to record the behavior of ZEN2 station. Figure 6(b) illustrates the smart sensor that is holding the three devices. One more important fact in the accelerometer campaign is that information was recorded at the same time than GPS and smartphone, providing a correct synchronization [37].

8.3. Smartphone Campaign. As illustrated earlier in Figure 4, the smartphone was fixed at the lowest plate of the mechanic structure for the smart sensor. The measured information from the accelerometer embedded in the smartphone was collected by an open access App [24]. This application can record acceleration and then send it to the user in the form of text file. The smartphone collected data at the same time than the GPS receiver and commercial accelerometer during 500 seconds, with a sampling rate of $400 \mathrm{~Hz}$.

8.4. Flow Chart Diagram of the Measurement Process. In summary, the data acquisition process of the bridge using the smart sensor is illustrated in Figure 7. Firstly, the accelerometer, smartphone, and GPS antenna are perfectly fixed to the location of ZEN2 station. The particular issues related to this location were previously discussed. Secondly, acceleration time histories are extracted from accelerometer and smartphone, respectively, and displacements from GPS receivers. As documented earlier, the data acquisition process is performed simultaneously using the three instruments. Thirdly, the apparent displacement in three directions (N-S, E-W, and vertical) is calculated using GPS. Then, semistatic and dynamic displacements are extracted using low- and high-pass filters, respectively. Fourthly, in order to study the dynamic characteristics of the Juarez Bridge, FFT and periodogram are plotted for the longitudinal, transversal, and vertical directions using raw acceleration of smartphone and accelerometer, respectively. Finally, to compare the extracting response capabilities of the three instruments, dynamic displacements reported by every device are used for the calculation of periodograms. The above discussion is clarified in the next section.

\section{Structural Response Collected by the Smart Sensor}

The structural performance of the Juarez Bridge is studied under normal service loading conditions in real time, i.e., the structure is evaluated during a loading condition that is experienced daily by users. In fact, a nonpermissible vibration in the vertical direction has been reported by users, and it has been documented in the literature by Vazquez-Becerra et al. [26]. To verify the undesirable behavior of this bridge, in this study, the authors decided to implement other devices during the SHM. Hence, the collected structural response is obtained simultaneously using GPS, accelerometer, and smartphone in real time, as a fused smart sensor.

9.1. Acceleration Time History. One measurement session of 500 seconds was performed using the three instruments under consideration. In terms of collected acceleration, Figure 8 illustrates the recorded response of the smartphone and accelerometer, respectively. Figures 8 (a)-8(c) illustrate the longitudinal, transversal, and vertical acceleration, respectively. Several observations can be made corresponding to Figure 8. It can be observed that acceleration in the vertical direction is greater than longitudinal and transversal directions. In terms of displacements, the same observation was reported by [26]. In addition, it can be noted in Figure 8(a) that in the longitudinal direction, the acceleration reported by smartphone and accelerometer is presenting some discrepancies. This issue may be justified because of the precision of commercial accelerometers in such a direction. However, this structural response does not represent a threat of safety because of the stiffness of the bridge in the longitudinal direction. In addition, it can be observed in Figure 8(c) that the critical component is associated to accelerations acting in the vertical direction. Conversely, in terms of accuracy, in Figures 8(b) and 8(c) is shown that acceleration for both smartphone and accelerometer is matching very closely. In general terms, Figure 8 demonstrates that acceleration recorded by smartphone and accelerometer can be positively comparable.

9.2. Semistatic Displacements Using GPS. Considering displacements obtained via GPS, semistatic displacements are 


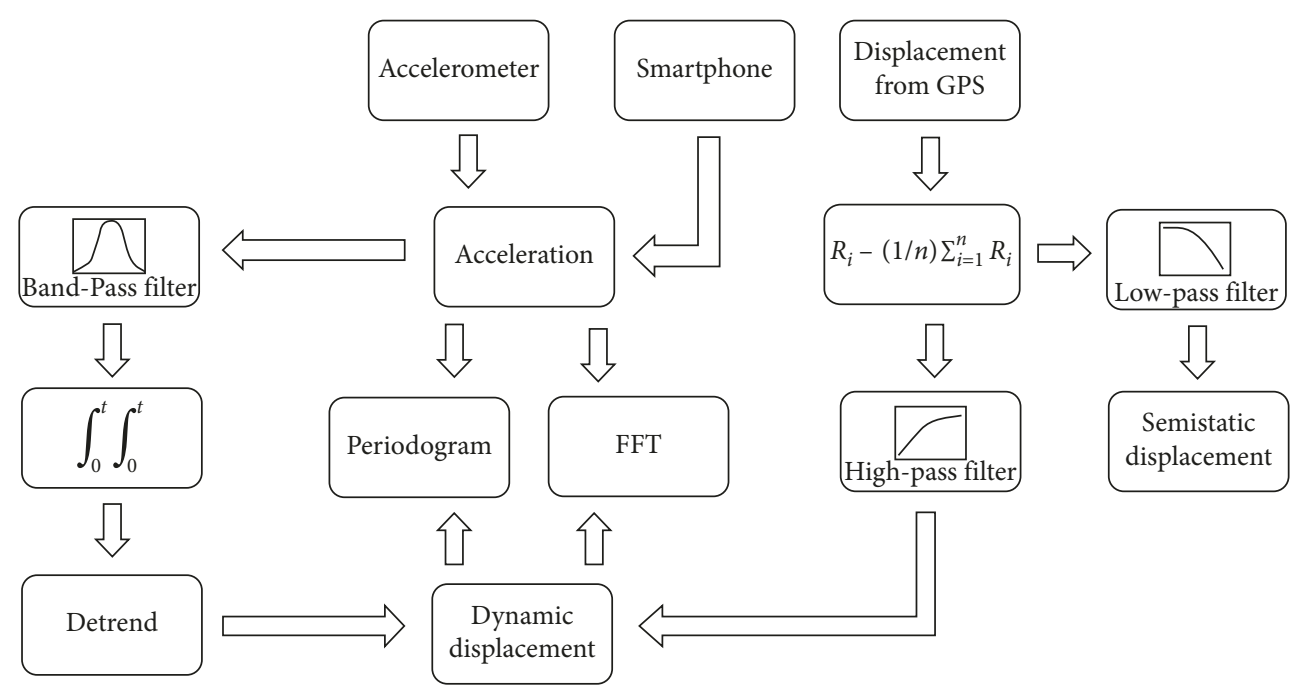

Figure 7: Flow chart of measurement process.

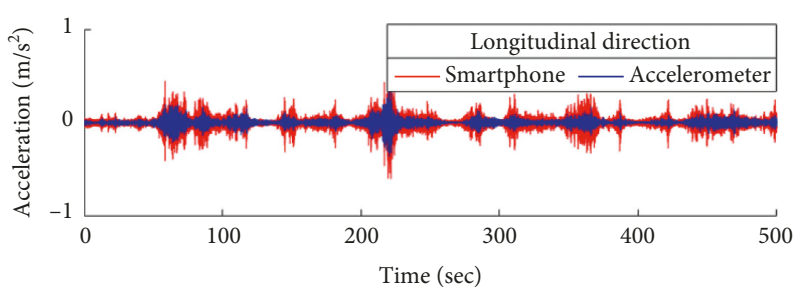

(a)

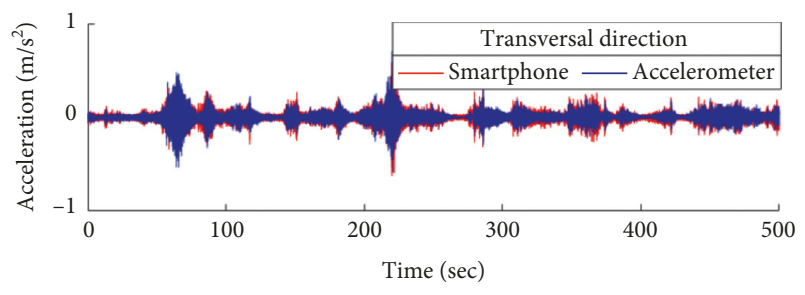

(b)

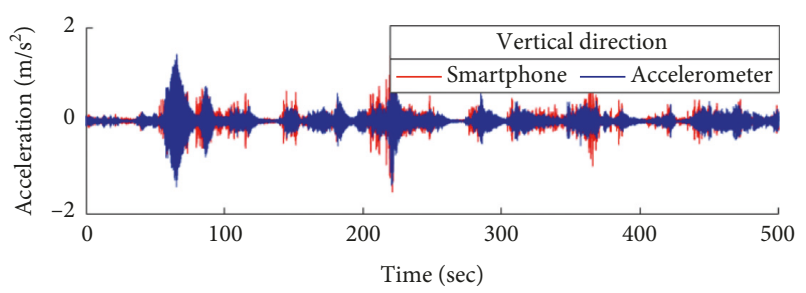

(c)

FIgURe 8: Acceleration time history.

extracted by applying a low-pass filter. Semistatic displacements corresponding to the bridge under consideration represent slow movements of the structure, they are reported in the N-S, E-W, and vertical direction in Figures 9(a)-9(c), respectively.

It can be observed in Figures 9(a) and 9(b) that smaller displacements are present in both N-S and E-W horizontal directions than those in the vertical one. This is illustrated in Figure 9(c), it is noted that considerable great displacements are reported in the vertical direction. Hence, in terms of semistatic displacements, it can be justified that the vertical component is presenting critical behavior. There exist cases of bridge studies with longer dimensions, such as the San Francisco-Oakland bay bridge that presented small displacements [38]. However, the analyzed bridge presented in this paper is a very particular case of study, which reports unusual behavior with respect to the vertical direction, comparing semistatic displacement with AASHTO deflection limits. That's why the authors of this paper decided to use it for the evaluation of the sensing capabilities of the proposed smart sensor.

9.3. Dynamic Displacements Using GPS. Movements related to short period component are represented by dynamic displacements (see Figure 10).

Dynamic displacements are obtained applying a highpass filter to GPS apparent displacements. Figure 10 illustrates dynamic displacements corresponding to the bridge. It can be noted in Figures 10(a) and 10(b) that dynamic displacements are relatively small for both N-S and E-W horizontal components. On the contrary, the vertical direction is presenting excessive displacements, and this is illustrated in Figure 10(c). One more time, critical displacements are appearing for the vertical component, but in this case with respect to dynamic displacements. 


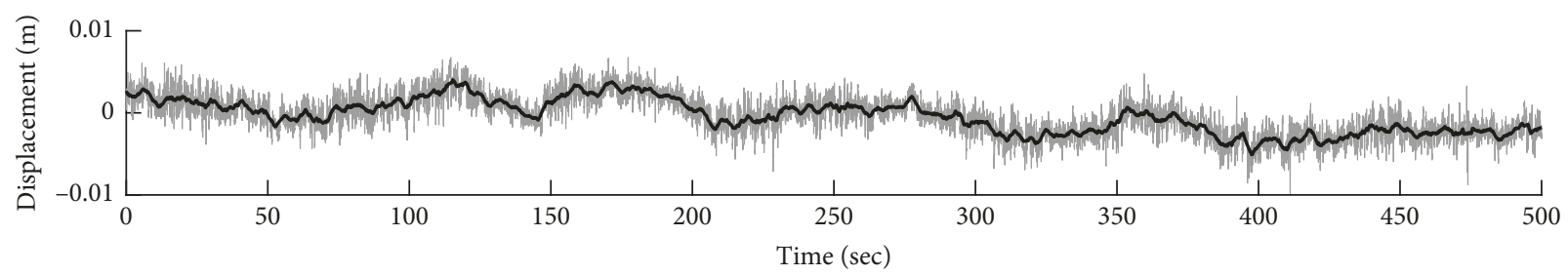

- Apparent displacement

— Semistatic displacement

(a)

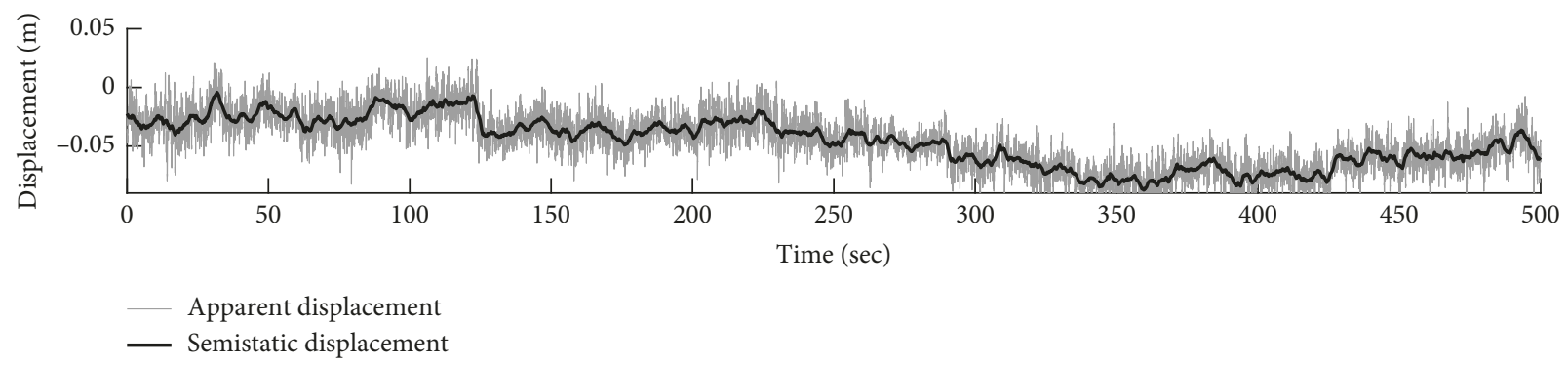

(b)

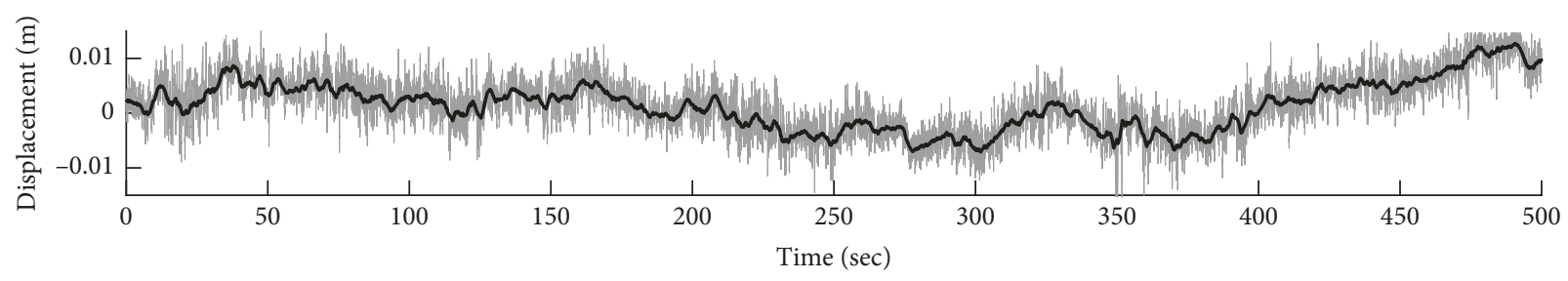

- Apparent displacement

— Semistatic displacement

(c)

Figure 9: Semistatic displacements in three directions: (a) N-S, (b) E-W, and (c) vertical.

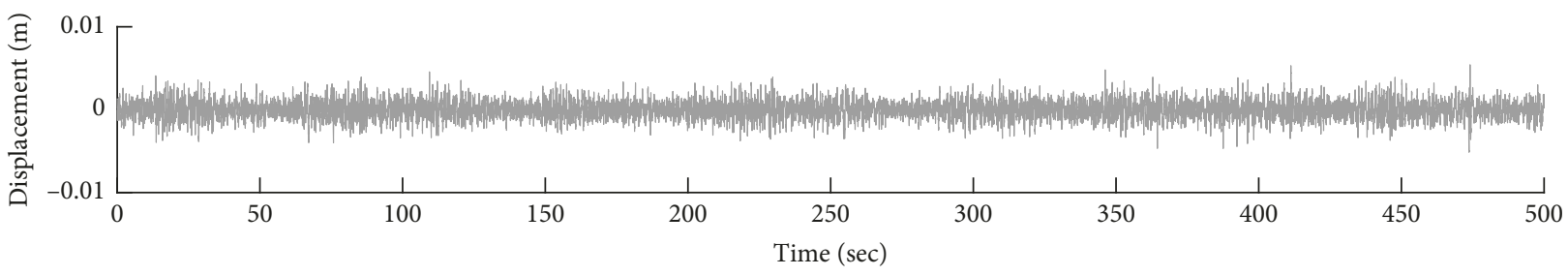

N-S

(a)

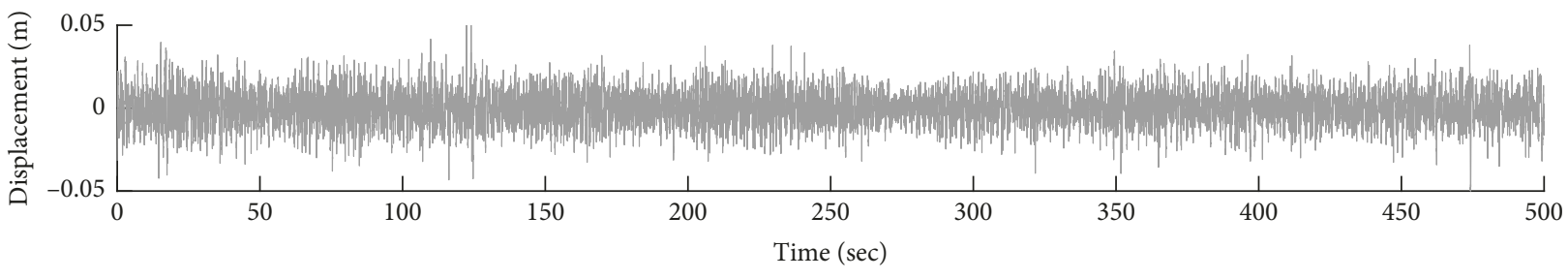

E-W

(b)

Figure 10: Continued. 


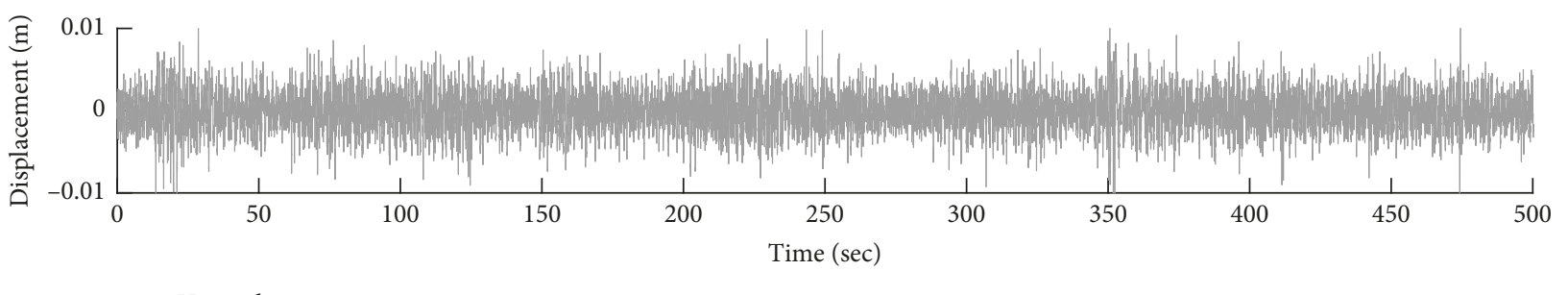

(c)

FIgURE 10: Dynamic displacements in three directions: (a) N-S, (b) E-W, and (c) vertical.

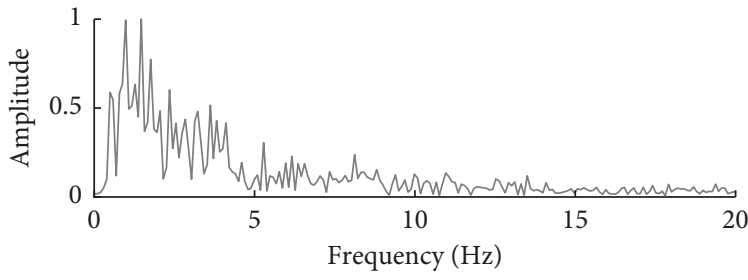

FFT

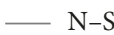

(a)

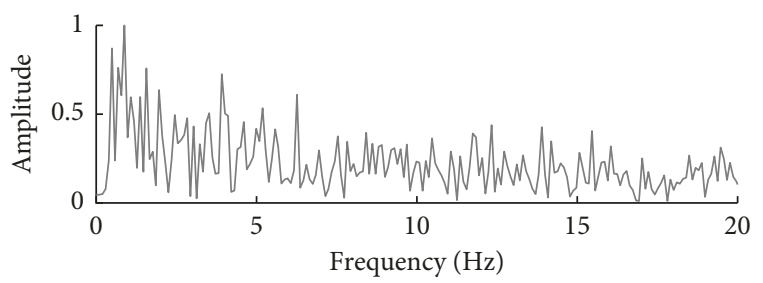

FFT

- E-W

(c)

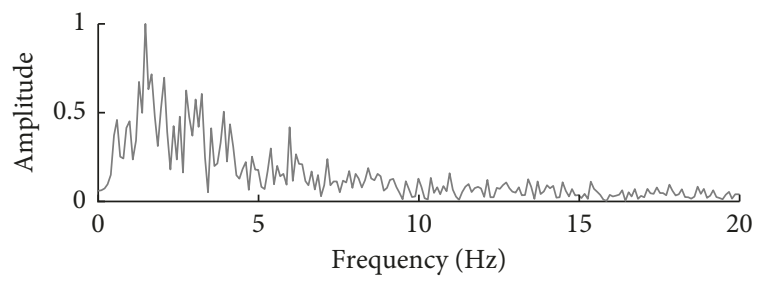

FFT

_ Vertical

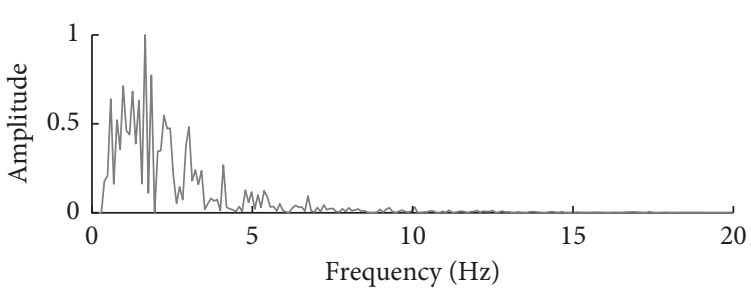

Periodogram

- N-S

(b)

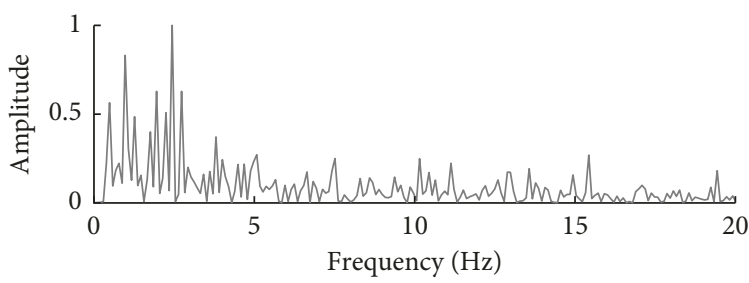

Periodogram

— E-W

(d)

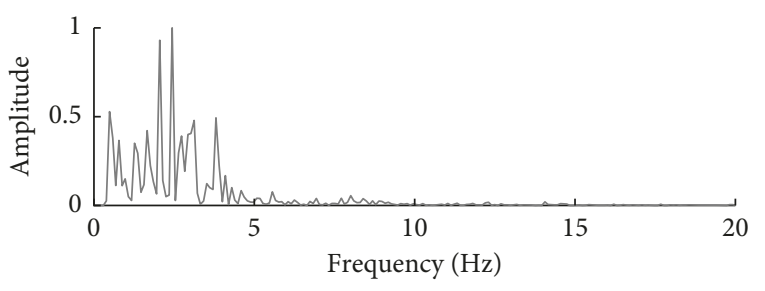

Periodogram

— Vertical

(e)

(f)

FIGURE 11: Dynamic frequencies in terms of normalized FFT and periodogram.

9.4. FFT and Periodogram Using GPS Dynamic Displacements. To identify structural dynamic characteristics of the structure under consideration, FFT and periodograms are calculated and plotted in frequency domain using dynamic displacements of GPS.

Figure 11 illustrates FFT and periodogram in the three GPS directions: N-S (Figures 11(a) and 11(b)), E-W (Figures 11(c) and 11(d)), and vertical (Figures 11(e) and 11(f)). To clarify the results, they are normalized with respect to their maximum amplitude value.
9.5. Acceleration Time History Using Accelerometer and Smartphone. Acceleration time histories corresponding to the longitudinal, transversal, and vertical direction of the bridge were earlier presented in Figure 8, considering both smartphone and accelerometer. In the longitudinal direction (see Figure 8(a)), it can be noted that there are some differences in the acceleration reported by smartphone and accelerometer; such discrepancies were justified earlier in Section 9.1. In the transversal (see Figure 8(b)) and vertical directions (see Figure 8(c)), it is observed that smartphone 

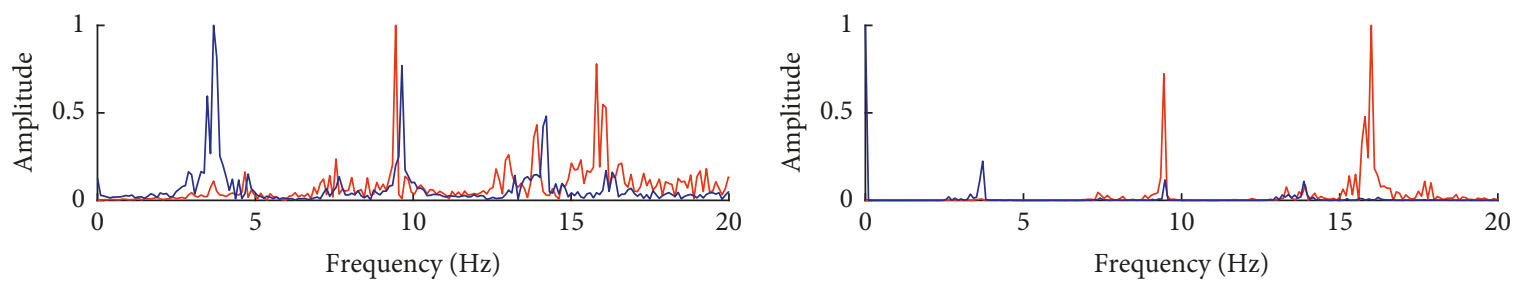

FFT_longitudinal direction
Smartphone
- Accelerometer

(a)

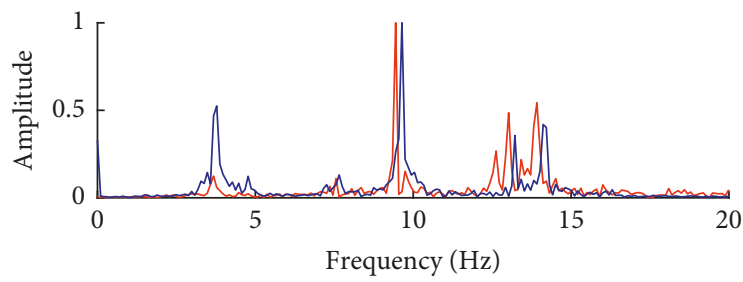

FFT - transversal direction

- Smartphone

- Accelerometer

(c)

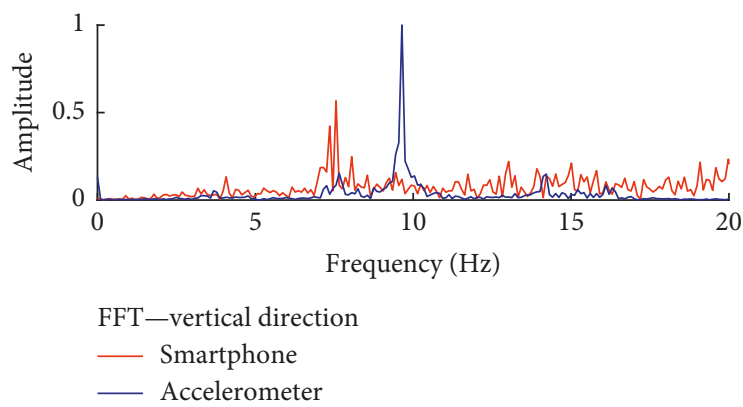

(e)

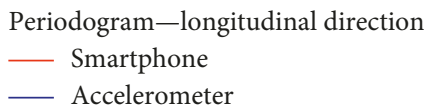

Periodogram-longitudinal direction

- Smartphone

_ Accelerometer

(b)

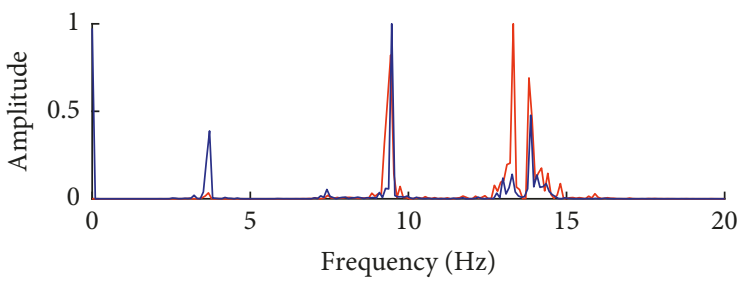

Periodogram-transversal direction

- Smartphone

_ Accelerometer

(d)

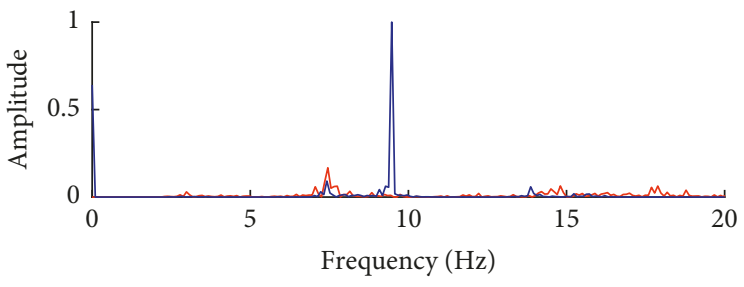

Periodogram-vertical direction

- Smartphone

- Accelerometer

Figure 12: Dynamic frequencies in terms of FFT and periodogram.

and accelerometer are recording very similar results. In time domain, these results are very promising. However, to complement them, results are changed to the frequency domain in terms of FFT and periodogram. This is discussed in the next section.

9.6. FFT and Periodogram Using Accelerometer and Smartphone Accelerations. In this section, results are studied in frequency domain. They are presented in terms of FFT and periodogram (see Figure 12).

Figure 12 summarizes the results related to FFT and periodogram corresponding to longitudinal, transversal, and vertical directions. It can be observed in Figure 12 that the frequency that contributes the most to the structural response may be located approximately at $9 \mathrm{~Hz}$. In addition, it is noted that FFT, in the longitudinal and transversal direction (see Figures 12(a) and 12(c)), is presenting the first frequency of interest at about $3.5 \mathrm{~Hz}$. Based on the results plotted in Figure 12 it can be stated that both smartphone and accelerometer are providing very accurate results with respect to each other. Hence, it may result practical to use smartphones for extracting dynamic characteristics of realscale bridges.

9.7. Comparison of Periodogram of GPS, Accelerometers, and Smartphones Using Dynamic Displacements. To compare data acquisition capabilities of smartphones, accelerometers, and GPS, the periodogram of them with respect to dynamic displacements in the vertical direction is illustrated in Figure 13. Only vertical direction is studied because it was demonstrated that this direction presented a critical behavior. It is noted, as reported in Figure 12, that the frequency that contributes the most to the structural response is located approximately at $9.37 \mathrm{~Hz}$, which is related to previous acceleration spectra. This frequency is well detected by the three instruments. Periodogram reported in Figure 13 is plotted considering dynamic displacements of smartphone, accelerometer, and GPS. Hence, the acceleration recorded by the smartphone and accelerometer, respectively, is converted to dynamic displacement by carrying out a 


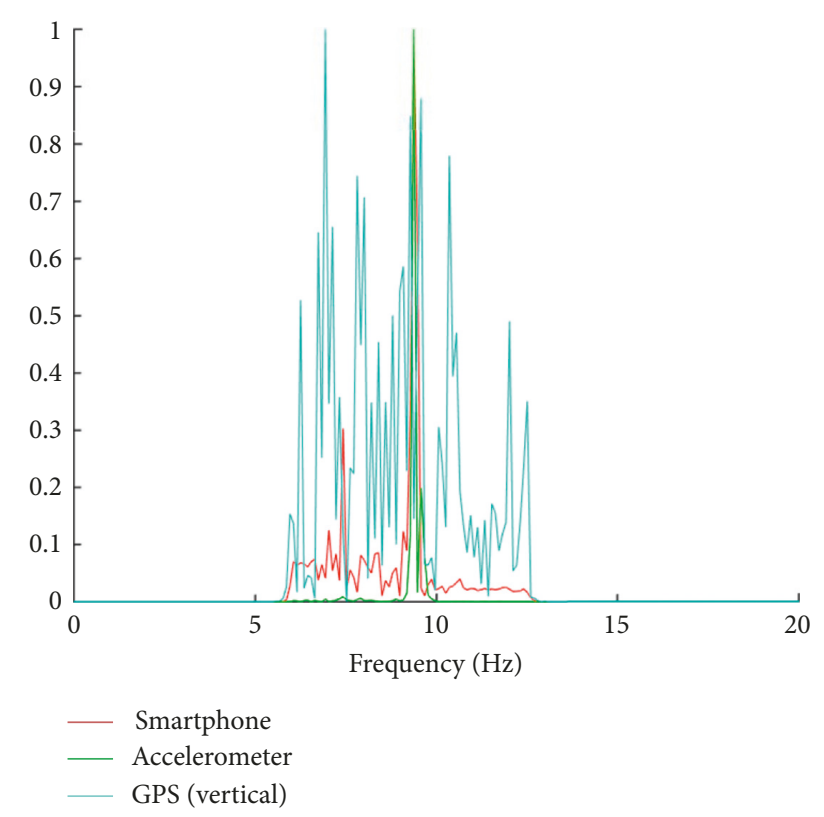

FIGURE 13: Dynamic frequencies in terms of periodogram (vertical direction).

double integration which may involve some discrepancies in obtaining displacements. In addition to numerical integration, some of the differences observed in Figure 13 may be associated with the applied filtering techniques to obtain dynamic displacements. On the other hand, GPS reports displacements directly, improving considerably data processing and interpretations. However, it is considered important to analyze the three periodograms from Figure 13 to have a better understanding about the coincidences of some frequencies that appear in two or more periodograms and it may be useful to have a better idea about de dynamic behavior of a bridge.

\section{Conclusions}

An alternative methodology to perform SHM on real-scale bridges is presented in this paper using GPS, an accelerometer, and a smartphone integrated in a fused smart sensor. Such sensor is validated for the SHM of complex structures as bridges under normal serviceability conditions in real time. A reinforced concrete bridge was studied by using the proposed smart sensor because it presented excessive vibration problems. The accuracy of this smart sensor was demonstrated comparing the gathered data from GPS, accelerometers, and smartphones in both time and frequency domain. Semistatic and dynamic displacements as well as accelerations demonstrated that there are critical performance problems on the analyzed bridge, particularly in the vertical direction. Since this critical behavior was reported by other scholars that performed the SHM with different devices, it can be stated that this smart sensor can be implemented for SHM on bridges in real time. In addition, it was demonstrated that the proposed smart sensor is adequate to perform SHM in real-scale bridges. Consequently, for the bridge under consideration, it was calculated that the fundamental frequency in the horizontal direction is located at $9 \mathrm{~Hz}$. Furthermore, it was detected the first frequency of interest around $3.5 \mathrm{~Hz}$. In order to improve frequency domain analysis, the proposed smart sensor methodology performs a periodogram algorithm over the vertical direction data to obtain a more accurate SHM. It was observed that there is a difference between GPS displacement data and accelerometer-smartphone based data. It can be justified due to the nature of each measurement methodology, where GPS base data comes from atmosphere and accelerometer-based data comes from a vibration sensor. Because of this, the noise nature of the aforementioned measurement devices is not the same due to its different measurement principle. On the other hand, it was observed a coincidence of the three instruments approximately at 9.37 Hz for the vertical component. Thus, it can explain the usefulness of fusing GPS, accelerometer, and smartphone information in order to discriminate undesired noise that can be generated by atmosphere disturbances of double integration error from acceleration data when it appears only in one device data. Finally, it can be stated that this paper contributed in understanding how GPS, accelerometers, and smartphones can be successfully fused in a smart sensor to perform SHM on real-scale bridges.

\section{Data Availability}

The data used to support the findings of this study are available from the corresponding author upon request.

\section{Disclosure}

Any opinions, findings, conclusions, or recommendations expressed in this paper are those of the authors and do not necessarily reflect the views of the sponsors.

\section{Conflicts of Interest}

The authors do not have any conflicts of interest to declare about this research.

\section{Acknowledgments}

The authors would like to thank Septentrio Inc. for providing the AsteRx-U Receiver and the PolaNt-x MF antenna used during the GPS data collection. This study is based on work partly supported by the Consejo Nacional de Ciencia y Tecnología (CONACYT-Mexico) as scholarships for the first and seventh author, respectively. In addition, the work is also supported by the Universidad Autónoma de Sinaloa (UAS).

\section{References}

[1] V. Ashkenazi and G. W. Roberts, "Experimental monitoring of the Humber bridge using GPS," Proceedings of the Institution of Civil Engineers-Civil Engineering, vol. 120, no. 4, pp. 177-182, 1997.

[2] W. S. Chan, Y. L. Xu, X. L. Ding, and W. J. Dai, “An integrated GPS-accelerometer data processing technique for structural 
deformation monitoring," Journal of Geodesy, vol. 80, no. 12, pp. 705-719, 2006.

[3] S. B. Im, S. Hurlebaus, and Y. J. Kang, "Summary review of GPS technology for structural health monitoring," Journal of Structural Engineering, vol. 139, no. 10, pp. 1653-1664, 2011.

[4] M. R. Kaloop and H. Li, "Monitoring of bridge deformation using GPS technique," KSCE Journal of Civil Engineering, vol. 13, no. 6, pp. 423-431, 2009.

[5] M. Kaloop, E. Elbeltagi, J. Hu, and A. Elrefai, "Recent advances of structures monitoring and evaluation using GPS-time series monitoring systems: a review," ISPRS International Journal of Geo-Information, vol. 6, no. 12, p. 382, 2017.

[6] X. Li, C. Rizos, L. Ge, Y. Tamura, and A. Yoshida, "The complementary characteristics of GPS and accelerometer in monitoring structural deformation," in Proceedings of the ION 2005 National Technical Meeting, Institute of Navigation (ION), San Diego, CA, USA, January 2005.

[7] P. Psimoulis, S. Pytharouli, D. Karambalis, and S. Stiros, "Potential of Global Positioning System (GPS) to measure frequencies of oscillations of engineering structures," Journal of Sound and Vibration, vol. 318, no. 3, pp. 606-623, 2008.

[8] C. Watson, T. Watson, and R. Coleman, "Structural monitoring of cable-stayed bridge: analysis of GPS versus modeled deflections," Journal of Surveying Engineering, vol. 133, no. 1, pp. 23-28, 2007.

[9] C. Xiong and Y. Niu, "Investigation of the dynamic behavior of a super high-rise structure using RTK-GNSS technique," KSCE Journal of Civil Engineering, vol. 23, no. 2, pp. 654-665, 2018.

[10] B. Hofmann-Wellenhof, H. Lichtenegger, and E. Wasle, GNSS-Global Navigation Satellite Systems: GPS, GLONASS, Galileo, and More, Springer Science \& Business Media, Vienna, Austria, 2007.

[11] M. Celebi, "GPS in dynamic monitoring of long-period structures," Soil Dynamics and Earthquake Engineering, vol. 20, no. 5-8, pp. 477-483, 2000.

[12] D. K. Kim, J. I. Kim, and M. Q. Feng, "Instrumentation of bridges for structural health monitoring," KSCE Journal of Civil Engineering, vol. 5, no. 3, pp. 231-242, 2001.

[13] G. Q. Wang, D. M. Boore, H. Igel, and X. Y. Zhou, "Some observations on colocated and closely spaced strong groundmotion records of the 1999 Chi-Chi, Taiwan, earthquake," Bulletin of the Seismological Society of America, vol. 93, no. 2, pp. 674-693, 2003.

[14] J. Hwang, H. Yun, S. K. Park, D. Lee, and S. Hong, "Optimal methods of RTK-GPS/accelerometer integration to monitor the displacement of structures," Sensors, vol. 12, no. 1, pp. 1014-1034, 2012.

[15] S. Dashti, J. D. Bray, J. Reilly, S. Glaser, A. Bayen, and E. Mari, "Evaluating the reliability of phones as seismic monitoring instruments," Earthquake Spectra, vol. 30, no. 2, pp. 721-742, 2014.

[16] M. Feng, Y. Fukuda, M. Mizuta, and E. Ozer, "Citizen sensors for SHM: use of accelerometer data from smartphones," Sensors, vol. 15, no. 2, pp. 2980-2998, 2015.

[17] X. Zhao, R. Han, Y. Ding et al., "Portable and convenient cable force measurement using smartphone," Journal of Civil Structural Health Monitoring, vol. 5, no. 4, pp. 481-491, 2015.

[18] X. Zhao, K. Ri, R. Han, Y. Yu, M. Li, and J. Ou, "Experimental research on quick structural health monitoring technique for bridges using smartphone," Advances in Materials Science and Engineering, vol. 2016, no. 1, pp. 1-14, 2016.

[19] Y. Ding, R. Han, H. Liu, S. Li, X. Zhao, and Y. Yu, "Bridge inspection and management system based on smart phone," in Proceedings of the ASME 2016 Conference on Smart
Materials, Adaptive Structures and Intelligent Systems, American Society of Mechanical Engineers, Stowe, VT, USA, September 2016.

[20] X. Zhao, R. Han, K. J. Loh, B. Xie, J. Li, and J. Ou, "Shaking table tests for evaluating the damage features under earthquake excitations using smartphones," in Proceedings of the Health Monitoring of Structural and Biological Systems XII, International Society for Optics and Photonics, Denver, CO, USA, May 2018.

[21] F. Moschas and S. Stiros, "Measurement of the dynamic displacements and of the modal frequencies of a short-span pedestrian bridge using GPS and an accelerometer," Engineering Structures, vol. 33, no. 1, pp. 10-17, 2011.

[22] M. R. Kaloop, "Bridge safety monitoring based-GPS technique: case study Zhujiang Huangpu bridge," Smart Structures and Systems, vol. 9, no. 6, pp. 473-487, 2012.

[23] G. W. Roberts, X. Meng, and A. H. Dodson, "Integrating a global positioning system and accelerometers to monitor the deflection of bridges," Journal of Surveying Engineering, vol. 130, no. 2, pp. 65-72, 2004.

[24] Accelerometer Analyzer App, 2017 , https://play.google.com/ store/apps/details?id=com.lul.accelerometer\&hl=es.

[25] X. Meng, "Real-time deformation monitoring of bridges using GPS/accelerometers," Doctoral dissertation, University of Nottingham, UK, 2002.

[26] G. E. Vazquez-Becerra, J. R. Gaxiola-Camacho, R. Bennett, G. M. Guzman-Acevedo, and I. E. Gaxiola-Camacho, "Structural evaluation of dynamic and semi-static displacements of the Juarez bridge using GPS technology," Measurement, vol. 110, no. 1, pp. 146-153, 2017.

[27] P. Psimoulis, I. Peppa, L. Bonenberg, S. Ince, and X. Meng, "Combination of GPS and RTS measurements for the monitoring of semi-static and dynamic motion of pedestrian bridge," in Proceedings of the 3rd Joint International Symposium on Deformation Monitoring, Vienna, Austria, April 2016.

[28] F. Moschas and S. Stiros, "Noise characteristics of highfrequency,short-duration GPS records from analysis of identical, collocated instruments," Measurement, vol. 46, no. 4, pp. 1488-1506, 2013.

[29] S. W. Smith, The Scientist \& Engineer's Guide to Digital Signal Processing, California Technical Publishing, San Diego, CA, USA, 1997.

[30] J. Wang, X. Meng, C. Qin, and J. Yi, "Vibration frequencies extraction of the forth road bridge using high sampling GPS data," Shock and Vibration, vol. 2016, Article ID 9807861, 18 pages, 2016.

[31] J. Yu, X. Meng, X. Shao, B. Yan, and L. Yang, "Identification of dynamic displacements and modal frequencies of a mediumspan suspension bridge using multimode GNSS processing," Engineering Structures, vol. 81, no. 1, pp. 432-443, 2014.

[32] E. Elbeltagi, M. Kaloop, and M. Elnabwy, "Monitoring and assessment of Mansoura railway steel bridge using RTK-GPS technique," in Proceedings of the 2nd Joint International Symposium on Deformation Monitoring, pp. 9-11, Nottingham, England, September 2013.

[33] N. Raziq, "GPS structural deformation monitoring: the midheight problem," Doctoral dissertation, University of Melbourne, Australia, 2008.

[34] S. S. Haykin, Adaptive Filter Theory, Pearson Education, India, 5th edition, 2014.

[35] L. H. Estey and C. M. Meertens, "TEQC: the multi-purpose toolkit for GPS/GLONASS data," GPS Solutions, vol. 3, no. 1, pp. 42-49, 1999. 
[36] G. W. Roberts, C. J. Brown, X. Meng, and P. R. B. Dallard, "Using GPS to measure the deflections and frequency responses of the London millennium bridge," in Bridge Design, Construction and Maintenance, Institution of Civil Engineers, London, UK, 2015, https://www.icevirtuallibrary.com/doi/10. 1680/bdcam.35935.0053.

[37] X. Meng, A. H. Dodson, and G. W. Roberts, "Detecting bridge dynamics with GPS and triaxial accelerometers," Engineering Structures, vol. 29, no. 11, pp. 3178-3184, 2007.

[38] D. McCallen, A. Astaneh-Asl, S. Larsen, and L. Hutchings, "Dynamic response of the suspension spans of the san Francisco-Oakland bay bridge," in Proceedings of the 8th U.S. National Conference on Earthquake Engineering, San Francisco, CA, USA, April 2006. 


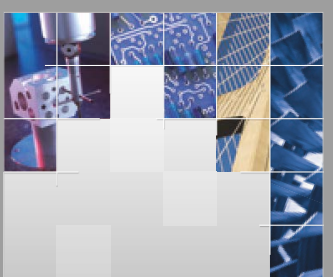

\section{Enfincering}
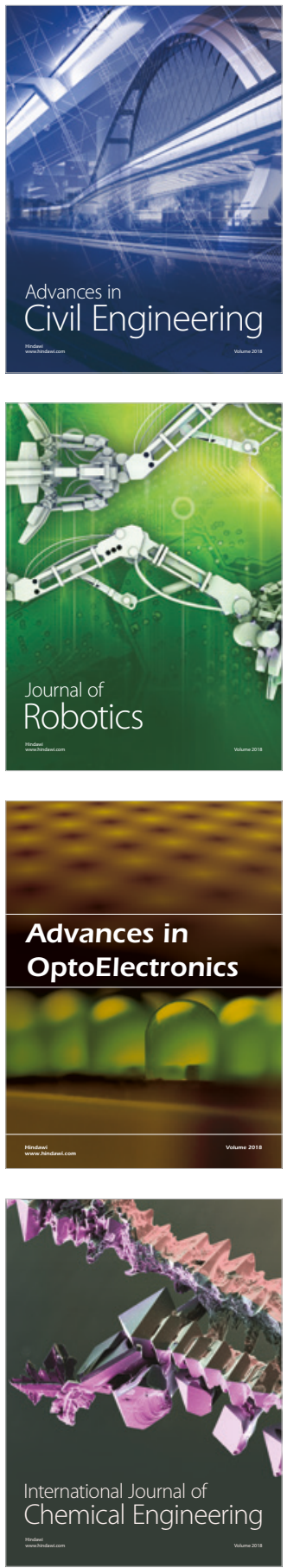

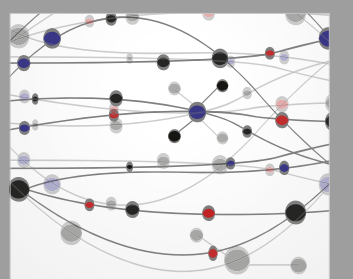

\section{Rotating \\ Machinery}

The Scientific World Journal

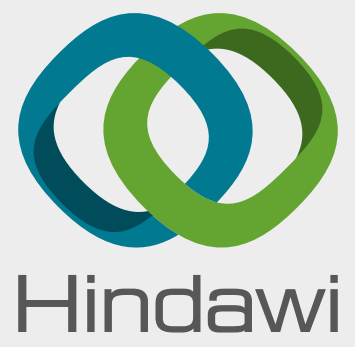

Submit your manuscripts at

www.hindawi.com
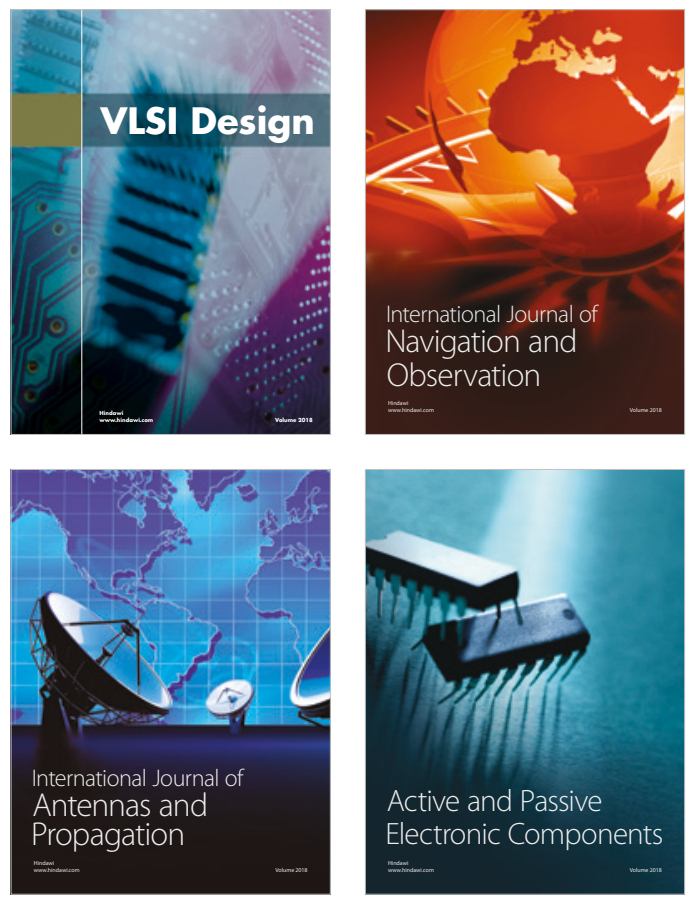
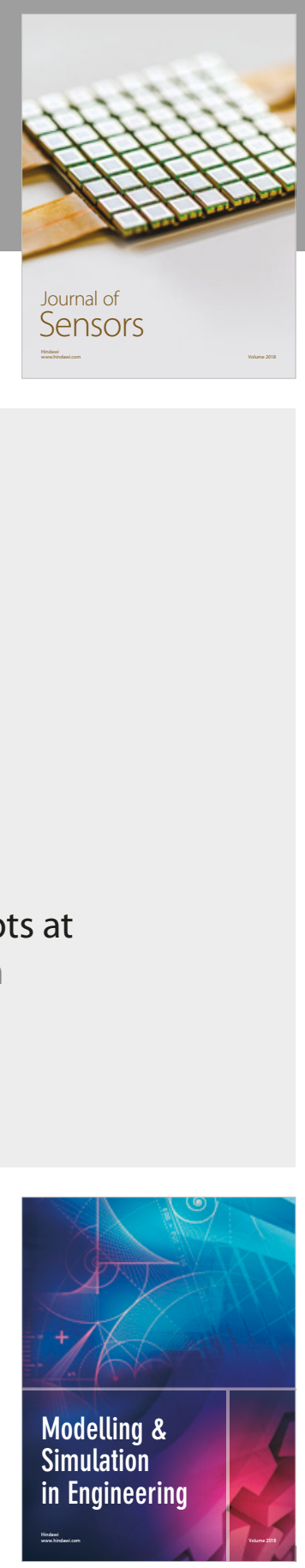

\section{Advances \\ Multimedia}
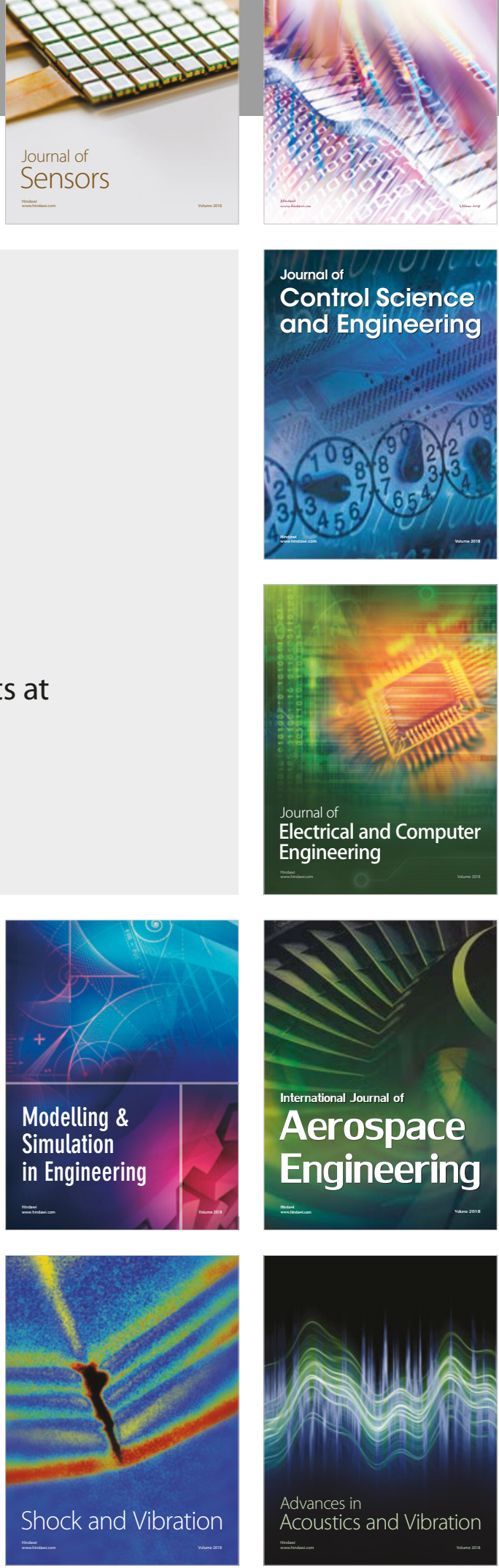